\title{
Formação de mestres em enfermagem na Universidade Federal de Santa Catarina: contribuições sob a ótica de egressos
}

Formation of masters in nursing at the Universidade Federal de Santa Catarina: contributions under post graduates' view Formación de maestros en enfermería en la Universidade Federal de Santa Catarina: contribuciones según la visión de los egresados

\section{Flávia Regina Souza Ramos', Vânia Marli Schubert Backes', Dirce Stein Backes', Dulcinéia Ghizoni Schneider', Gleide Pinheiro', Maria Terezinha Zeferino', Patríca Kuerten Rocha'}

'Universidade Federal de Santa Catarina. Departamento de Enfermagem. Programa de Pós-graduação em Enfermagem. Florianópolis, SC

Submissão: 15/09/2009

Aprovação: 19/08/2009

\section{RESUMO}

O estudo objetivou conhecer as percepções de egressos do Curso de Mestrado em Enfermagem do Programa de Pós-graduação em Enfermagem da Universidade Federal de Santa Catarina acerca das contribuições de sua formação para o trabalho profissional. Estudo de abordagem Qualitativa, utilizou Questionário aplicado a mestres em enfermagem egressos do curso (últimos 5 anos), totalizando 88 informantes. A análise apresentou elementos da ótica dos egressos Quanto a uma avaliação geral do curso, Quanto às contribuições do mesmo para a mudança no trabalho e Quanto a avaliação média dos seus objetivos. O mestrado em enfermagem mostrou-se um espaço reconhecido e valorizado de construção e consolidação de um conhecimento inovador frente aos desafios Que se impõem para uma nova configuração do fazer em saúde cotidianamente.

Descritores: Enfermagem; Avaliação Educacional; Educação em Enfermagem.

\section{ABSTRACT}

The objective of the study is to know the perceptions of graduates of the Masters Course in Nursing at the Universidade Federal de Santa Catarina with its contributions on formation as regards to professional work. That was a study of Qualitative approach, using a Questionnaire applied to the egresses of master degree program (last 5 years), totaling 88 informants. Analysis presented elements of the graduates lent in relation to the general evaluation of the course, as related to the same contributions for change at work and the medium evaluation of its objectives. The masters degree program shows a space and the valorization of construction and consolidation of an innovative knowledge that can faces challenges that might occur to a new configuration of making health daily.

Key words: Nursing; Educational Evaluation; Education Nursing.

\section{RESUMEN}

El estudio tuvo como objetivo conocer las percepciones de los egresados de la Maestría en Enfermería de la Universidade Federal de Santa Catarina acerca de las contribuciones de su formación para el trabajo profesional. Estudio de aboerdage cualitativa, con la utilización de un cuestionario dirigido a los maestros egresados en los últimos cinco años, para un total de 88 informantes. El análisis presentó elementos en relación a evaluación general de la Maestría, así como de las contribuciones de la misma para el cambio en el trabajo y la evaluación de sus objetivos. La maestría en enfermería se presentó como un espacio reconocido y valorizado para la construcción y consolidación de conocimientos innovadores ante los desafíos para una nueva configuración del Quehacer en salud.

Descriptores: Enfermería; Evaluación Educacional; Educación en Enfermería. 


\section{INTRODUÇÃO}

No meio acadêmico é reconhecida a importância de processos permanentes de avaliação da formação profissional em todos os seus níveis, por considerar Que tais processos são instrumentais para se obter visões mais consistentes sobre a Qualidade dos produtos do trabalho institucional, ou seja, sobre o alcance dos objetivos e a função social de instituições e cursos. Diversas são as informações necessárias para alimentar um processo de avaliação, além de serem também variados os possíveis participantes e interessados, envolvendo desde gestores até beneficiários ou usuários. Por diferentes argumentos mais e mais se reafirma a complexidade de processos avaliativos e se oferecem diferentes marcos teórico-metodológicos para seu desenvolvimento.

Independente do uso de planos e métodos específicos, a avaliação é elemento essencial de QualQuer ação social, na medida em Que subsidia a crítica e a tomada de posição com informações sobre o funcionamento de determinado programa, serviço ou ação, tanto em termos de resultados e impactos, como de operatividade e processos aplicados, Que poderão indicar novas demandas, metas ou estratégias para a Qualidade desejada.

No caso de processos avaliativos Que se referem a programas de pós-graduação é preciso considerar, pelo menos, duas importantes dimensões não excludentes e cooperantes entre si: a avaliação externa e a avaliação interna. A avaliação interna envolve a coordenação, o planejamento e a tomada de decisão, vistos como atividades contínuas e inerentes ao próprio trabalho em andamento, não apenas como etapa estanque e temporalmente isolada do restante da dinâmica de um curso. lá a avaliação externa de programas de pós-graduação é desenvolvida no cenário brasileiro pela Coordenação de Aperfeiçoamento de Pessoal de Nível Superior (CAPES) com comissões externas. Recentemente, incluiu na avaliação externa, os egressos. Este será o enfoque tomado neste estudo, Qual seja, dar voz aos egressos.

Ao ter como foco a sistematização de subsídios para a coordenação acadêmica e para a análise do processo de formação em desenvolvimento, o presente estudo parte da premissa de Que o Programa de Pós-graduação em Enfermagem da Universidade Federal de Santa Catarina - PEN/UFSC dispõe de diversos elementos de avaliação, contudo, deve ser promovida a discussão sobre o Que tais elementos fornecem em termos de informações, a busca por novos subsídios, o cotejamento de diferentes aspectos e, finalmente, a proposição de interpretações sobre tendências e perspectivas. Um dos elementos importantes para análise do impacto deste programa é conhecer a perspectiva dos egressos Quanto a sua experiência durante e após o curso.

Mesmo sem o caráter de um estudo avaliativo, é importante Que sejam considerados elementos contextuais do acervo de informações disponíveis, Que se referem ao Sistema Nacional de Avaliação da Pós-graduação e à própria trajetória histórica do PEN/ UFSC, mas especificamente de seu Curso de Mestrado, objeto deste estudo. Assim, identificando as diretrizes da atual Política Nacional da Pós-graduação, delineando o panorama da formação de mestres no Brasil e reconhecendo a trajetória do Curso de Mestrado em Enfermagem do PEN/UFSC, estabelecemos como objetivo deste estudo conhecer as percepções de egressos do Curso de Mestrado em Enfermagem do PEN-UFSC acerca das contribuições de sua formação para o trabalho profissional.

\section{Panorama da Pós-graduação no Brasil}

O programa de pós-graduação, no Brasil, surgiu na década de 60 e expandiu-se, mais precisamente, a partir da década de 90 , pela necessidade de Qualificar docentes para atender as demandas de expansão do ensino superior e ampliar a formação e capacitação de novos pesquisadores ${ }^{(1)}$. O CNPQ -Conselho Nacional de Desenvolvimento Científico e Tecnológico e a CAPES, órgãos criados no ano de 1951 , foram essenciais para a institucionalização e regulamentação da pesQuisa no Brasil através da criação da Política Nacional de Pós-graduação.

Nessa perspectiva, o Ministério da Educação e Cultura - MEC elaborou em 1975, o I Plano Nacional de Pós-Graduação - PNPG. As diretrizes previam a institucionalização do sistema, consolidandoo como atividade regular no âmbito das universidades e garantindoIhe financiamento estável ${ }^{(2)}$. O II PNPG, editado em 1982(3), priorizou a formação de recursos humanos Qualificados para as atividades docentes, de pesquisa e técnicas visando o atendimento dos setores público e privado, enfatizou a importância da avaliação, da participação da comunidade científica e do desenvolvimento da pesquisa científica e tecnológica. Em 1986 foi apresentado o III PNPG, tendo como principal ênfase o desenvolvimento da pesQuisa pela universidade e a integração da pós-graduação ao sistema de ciência e tecnologia ${ }^{(4)}$. O IV PNPG, consolidado em $1998^{(5)}$, estava baseado na avaliação do desempenho da CAPES, na expansão da pós-graduação em relação ao crescimento das áreas e deseQuilíbrios regionais, nos financiamentos e na atenção à demanda por estudos profissionalizantes, entre outros princípios.

O V PNPG, o Qual compreende o período de 2005-2010, tem como um dos seus objetivos fundamentais uma expansão do sistema de pós-graduação Que leve a expressivo aumento do número de pós-graduandos requeridos para a Qualificação do sistema de ensino superior do país, do sistema de ciência e tecnologia e do setor empresarial ${ }^{(6)}$.

Destacamos dentro das Diretrizes Gerais dos PNPG a avaliação, foco deste estudo, a Qual deve estar baseada na Qualidade e excelência dos resultados, na especificidade das áreas de conhecimento e no impacto dos resultados na comunidade acadêmica e empresarial e na sociedade ${ }^{(6)}$.

O Sistema Nacional de Avaliação da Pós-graduação integra todos os programas e cursos de Pós-graduação no Brasil; foi implantado pela CAPES em 1976 e hoje desempenha um papel reconhecido por sua relevância no desenvolvimento da pós-graduação e da pesquisa científica e tecnológica no Brasil. A Avaliação dos Programas, segundo a CAPES, compreende o acompanhamento anual e a avaliação trienal do desempenho, visando estabelecer o padrão de Qualidade exigido dos cursos de mestrado e de doutorado, contribuindo para o aumento de sua eficiência, o atendimento das necessidades nacionais e regionais de formação de recursos humanos de alto nível e para as definições de políticas na área.

Os dados da pós-graduação brasileira indicam Que todas as áreas do conhecimento apresentaram crescimento expressivo ao longo dos anos. O número de cursos recomendados pela CAPES era $673 \mathrm{em}$ 1976, 2.993 em 2004 e 3.855 em 2008. O Sistema Nacional de Pós-Graduação(7), em junho de 2008, possui 2.581 Programas, responsáveis por 2.319 cursos de mestrado $(60,1 \%), 1.312$ de 
doutorado (34,0\%) e 224 de mestrado profissionalizante (5,9\%). A maior área é a da saúde, como 434 programas. Enquanto em 1987, em todas as áreas de conhecimento, se titularam 3.647 mestres e 868 doutores, esse número subiu para 32.899 e 9.919 , respectivamente, em 2007. Atualmente conta com 51.000 bolsistas e 50.509 docentes. Apesar desta expressiva expansão, com impacto importante na produção científica brasileira no cenário mundial, o desenvolvimento da pós-graduação continua a demandar investimentos ${ }^{(8)}$.

\section{A Pós-Graduação em Enfermagem no Brasil e o Programa de Pós-Graduação em Enfermagem/UFSC}

Com a aprovação da Lei $5.540^{(9)}$ Que instituiu a Reforma Universitária Brasileira ao final da década de 1960, observou-se o interesse por implantação de cursos de pós-graduação na área de enfermagem com vistas a Qualificar docentes para apoiarem a expansão do ensino da enfermagem em nível de graduação recomendado ${ }^{(1)}$.

Desse modo, segundo a CAPES, o primeiro programa de pósgraduação strict-sensu, na modalidade de mestrado, foi implantado em 1972, na Escola de Enfermagem Ana Nery, da Universidade Federal do Rio de laneiro, e posteriormente em 1973 e 1975 nas Escolas de Enfermagem da Universidade de São Paulo em São Paulo e em Ribeirão Preto, respectivamente ${ }^{(10)}$.

A partir daí observou-se um gradativo aumento de pósgraduações strictu-sensu em enfermagem, sendo Que o primeiro doutorado foi autorizado em 1981 na Escola de Enfermagem da Universidade de São Paulo-SP.

Atualmente, o Brasil conta com 33 programas de pós-graduação strictu-sensu na área da enfermagem, dos Quais 12 são de mestrado e doutorado, 19 de mestrado ( 17 acadêmicos e 2 profissionalizantes) e 2 de doutorado ${ }^{(10)}$, conforme distribuição apresentada na tabela abaixo.

As três últimas décadas, notadamente as de 1980 e 1990 foram marcadas pela expansão de cursos e programas de pós-graduação em enfermagem, entretanto, observa-se Que as regiões Sul e Sudeste detêm o maior número de cursos em nível de mestrado, sendo que a Sudeste se destaca em número de doutorados.

O PEN/UFSC, constituído pelos Cursos de Mestrado e Doutorado, é reconhecido pela CAPES, e ao completar trinta anos de existência, em 2006, consolida-se enquanto Programa de excelência, titulando profissionais em nível de mestrado e de doutorado, não só da região sul, como também de outras regiões do Brasil e da América Latina.

Alguns indicadores merecem destaque pelos avanços significativos obtidos, dentre eles, a Qualificação do corpo docente, a produtividade, a diminuição do tempo de titulação e a internacionalização. No Que diz respeito à produtividade, de 1976 até setembro de 2007, foram titulados 470 Mestres e 143 Doutores.

O Curso de Mestrado em Saúde do Adulto, criado em 1976, foi submetido a avaliações periódicas, resultando, em 1986, na mudança da Área de Concentração, para Mestrado em Assistência de Enfermagem. A perspectiva adotada foi de aplicação e avaliação de referenciais teóricos na prática de Enfermagem, resultando em um impacto social positivo(ll).

Com a criação da Rede de Pós-Graduação em Enfermagem da
Região Sul (REPENSUL), em 1992, iniciou-se parcerias entre a UFSC e as universidades UFPR, UFSM, FURG, UFPEL, UFRGS, implantando-se a modalidade de Mestrado Expandido. Em 1996, foi introduzido o Mestrado Interinstitucional (MINTER) e os reflexos do trabalho de expansão do Programa em prol do desenvolvimento da Enfermagem da Região também se expressaram pela criação de seis novos Programas de Pós-Graduação (UFRGS em 1998, FURG em 2001, UFPR em 2002, UEM em 2003, UFSM em 2006 e UFPEL em 2007) e pela elaboração de projetos para implantação de Programas de Pós-Graduação em outras Universidades da Região ${ }^{(11)}$.

O investimento crescente na formação de mestres e doutores tem sido uma estratéria no intuito de contribuir para o desenvolvimento de uma prática profissional voltada para a busca de soluções relativas aos problemas de saúde vivenciados pela população brasileira ${ }^{(1)}$.

A partir de 2002, o curso passa a dirigir-se a profissionais de Enfermagem e de saúde, tendo como objetivo geral "promover o repensar das práticas de Enfermagem e de saúde, com base em referenciais teórico-metodológicos, apresentando contribuições crítico-inovadoras para o cuidado no processo de viver humano, e como objetivos específicos propor, aplicar e avaliar projetos assistenciais com base em reflexões críticas sobre as práticas de Enfermagem e de saúde; analisar de forma crítica os resultados de pesquisas com vistas a sua utilização no ensino, na gestão e na assistência em saúde no âmbito local, regional, nacional e internacional; desenvolver conhecimentos e habilidades de pesquisa na área de Enfermagem e de saúde; instrumentalizar os profissionais para a participação crítica no redirecionamento e/ou fortalecimento de políticas e práticas de Enfermagem e de saúde ${ }^{(1)}$.

\section{MÉTODO}

Tratou-se de um estudo de natureza Qualitativa, tendo como sujeitos mestres em enfermagem egressos do curso, Que concluíram sua formação entre janeiro de 2003 e setembro de 2007 (últimos 5 anos), totalizando 88 egressos. O método de coleta de dados foi um Questionário do tipo auto-aplicável, composto por dados de identificação e Questões abertas e fechadas, enviado via correio eletrônico, sendo utilizado o cadastro do PEN/UFSC para acesso aos endereços dos egressos, no período de outubro a dezembro de 2007. No estudo, os egressos foram codificados como P (participante) e o número de acordo com a seeüência de envio do Questionário (P3).

Os dados coletados foram submetidos à técnica de análise de conteúdo de Bardin ${ }^{(12)}$, considerando os estágios de pré-análise, constituição do corpus, seleção das unidades de análise, classificação, codificação e categorização e os resultados apresentados em gráficos e Quadros analisados descritivamente.

Atendendo às normas Que regem pesquisas Que envolvem seres humanos, notadamente a resolução 196/96 do Conselho Nacional de Saúde, o projeto foi submetido e aprovado pelo Comitê de Ética e Pesquisa da UFSC (Processo no 313/2007) e os participantes expressaram sua adesão por meio de Termo de Consentimento Livre e Esclarecido.

\section{RESULTADOS E DISCUSSÃO}

O presente trabalho se propôs levantar percepções de egressos 
do Curso de Mestrado (2002-2007) do Programa de PósGraduação em Enfermagem da Universidade Federal de Santa Catarina, acerca das contribuições de sua formação para o trabalho profissional. O Quadro I ilustra a percepção ou avaliação geral dos egressos do Curso de Mestrado, em relação aos aspectos: currículo do curso, carga horária do curso e disciplinas, disciplinas obrigatórias, disciplinas optativas, seqüência de oferecimento das disciplinas, interação entre as disciplinas, corpo docente, interdisciplinaridade do corpo docente, estrutura física e de apoio ao ensino, grupos de pesquisa e oportunidades de vivência acadêmica.

Os egressos, de modo geral, consideram como excelentes ou muito bons à maioria dos aspectos anteriormente expostos. Destacam-se, nessa avaliação, a Qualificação e competência do corpo docente e as oportunidades de vivência acadêmica Que, do ponto de vista dos participantes da pesquisa, deve ser estimulada e incrementada. As falas refletem estas percepções ao mencionarem:
“Apenas, gostaria de parabenizar o corpo docente e técnico administrativo da PEN/UFSC pelo trabalho realizado, mesmo sabendo das dificuldades que passam as instituições publicas de ensino" (PI).

"Embora a característica do Mestrado seja, na minha concepção, de um Mestrado Profissionalizante, creio Que os conhecimentos teórico-filosóficos adQuiridos possibilitaram enxergar novas modalidades de saber e fazer saúde, enfermagem e cuidado" (P2).

“Porque não nos limitamos a um só conhecimento, o mestrado nos mostra como refletir e modificar uma pratica profissional, seja no âmbito coletivo ou individual" (P3).

O Quadro 2 retrata as contribuições do Curso de Mestrado para a mudança no trabalho dos egressos. As manifestações dos informantes foram agrupadas de acordo com as categorias

\begin{tabular}{|c|c|c|c|c|c|c|}
\hline Variáveis analisadas & Excelente & Muito bom & Bom & Regular & Ruim & Péssimo \\
\hline Currículo do curso & 10 & 11 & 6 & 0 & 1 & 0 \\
\hline Carga horária e discipinas & 11 & 7 & 7 & 2 & 0 & 0 \\
\hline Disciplinas obrigatórias & 11 & 10 & 5 & 0 & 1 & 0 \\
\hline Disciplinas optativas & 7 & 11 & 8 & 2 & 0 & 0 \\
\hline Sequência de oferecimento & 9 & 9 & 9 & 2 & 0 & 0 \\
\hline Interação das disciplinas & 3 & 10 & 9 & 2 & 2 & 0 \\
\hline Corpo docente & 14 & 8 & 4 & 0 & 0 & 0 \\
\hline Interdisciplinaridade & 8 & 7 & 8 & 3 & 1 & 0 \\
\hline Estrutura física & 6 & 12 & 8 & 1 & 0 & 1 \\
\hline Grupos de pesquisa & 10 & 11 & 4 & 1 & 0 & 0 \\
\hline Oportunidades de vivências & 6 & 4 & 13 & 1 & 1 & 0 \\
\hline
\end{tabular}

Quadro 1. Avaliação geral do Curso de Mestrado do PEN-UFSC na ótica dos egressos. Florianópolis, 2007.

\begin{tabular}{|c|c|}
\hline Contribuições & Total \\
\hline Ampliação de conhecimentos e saberes & 4 \\
\hline Abertura para atuação como docente & 3 \\
\hline Novas possibilidades de emprego & 2 \\
\hline ReQuisito para inscrição em concurso público & 2 \\
\hline Assumiu coordenação & 1 \\
\hline Realização pessoal/Realização de um sonho & 2 \\
\hline Atuação em projetos de pesQuisa financiados & 1 \\
\hline Atendimento à exigência institucional & 1 \\
\hline Estímulo para aliar peseuisa à assistência & 1 \\
\hline Salário diferenciado & 1 \\
\hline Respaldo para interferir em situações complexas & 1 \\
\hline Não houve mudança & 1 \\
\hline Não responderam & 9 \\
\hline
\end{tabular}

Quadro 2. Contribuições do Curso de Mestrado PEN/UFSC para a mudança no trabalho dos egressos. Florianópolis, 2007. 
apresentadas nesse Quadro e demonstram as possibilidades de uma reestruturação político/acadêmica a partir de uma análise sobre as contribuições do curso no cotidiano profissional dos egressos.

É consenso Que a Qualificação para atuação como docente e como pesquisador ainda se constitui um dos principais focos dos cursos de pós-graduação Stricto-Sensu no Brasil, entretanto nesse estudo se verificou uma significativa inserção de profissionais Que atuam no âmbito do Sistema Único de Saúde (SUS) e pelas falas dispostas a seguir, é possível observar a ocorrência de mudanças significativas no cotidiano profissional dos egressos do curso avaliado.

"Pude agregar à minha formação, como fisioterapeuta, toda uma nova forma de assistir o ser humano e de transformar a reflexão do fazer em um novo fazer, ainda melhor e mais humano. Como professora, posso ter competência para trabalhar com pesquisa Qualitativa com mais propriedade". (P4)

“Em relação ao meu aprendizado, posso dizer que me tornei uma pessoa mais reflexiva e mais crítica e competente em meu ser e fazer. Além disso, o mestrado me despertou o interesse por projetos assistenciais e investigativos. Hoje os projetos representam uma constante do meu trabalho. Tudo o Que faço é inicialmente planejado e avaliado". (P5)

"Uma nova forma de assistir o ser humano e de transformar a reflexão do fazer em um novo fazer, ainda melhor e mais humano... Competência para trabalhar com pesquisa Qualitativa com mais propriedade". (P6)

Um Curso de Pós-Graduação na área de saúde tem uma influencia importante no contexto social no Qual está inserido, pois, além das exigências da Lei de Diretrizes e Bases da Educação( ${ }^{(13)}$, as configurações da Lei Orgânica da Saúde ${ }^{(14)}$ e a Lei Complementar $8142 / 1992^{(15)}$ convergem para a necessidade de desenvolvimento de pesquisas no âmbito do SUS como forma de aproximar a oferta de serviços e ações de saúde das necessidades apresentadas pela demanda.

Aliado a esses fatores, nos últimos anos convivemos com uma rápida modernização na estrutura e formação do conhecimento e no aprimoramento de técnicas e procedimentos no campo da saúde, exigindo do profissional um olhar investigativo, reflexivo e crítico no âmbito de sua atuação profissional. Assim, é primordial Que os conteúdos acessados e elaborados no decorrer de um curso de mestrado sejam propulsores de processos reflexivos Que impulsionem a capacidade e a vontade de se envolver com o entorno social no Qual está inserido, no intuito de estabelecer vínculos entre a prática profissional e a dinâmica social. A fala abaixo nos remete a essas reflexões

"O Mestrado me fez repensar sobre a vida, a profissão, podemos ver Que há outras formas de se fazer a assistência e a própria docência em saúde". (P7)

É preciso reconhecer, no entanto, Que as possibilidades de mudanças nesses contextos não estão vinculadas apenas a capacitação e a vontade do profissional, mas também ao envolvimento político/institucional Que contribua para estimular uma revitalização constante dos conhecimentos necessários ao desenvolvimento do processo de trabalho diante dos novos desafios Que se impõem a uma nova configuração do fazer saúde cotidianamente, exigindo uma constante interação entre a vontade pessoal e a vontade social.

$\mathrm{Na}$ fala a seguir, percebemos Que a informante se deparou com as dificuldades impostas pela instituição na Qual atuava, levando-a a optar pela mudança de emprego.

"Para mim o mestrado foi ótimo, mas na instituição de ensino pouco mudou, possivelmente porque fora desencadeado em nós expectativas Que não foram cumpridas e, desta forma, agora estou buscando um novo emprego, penso inclusive em mudar de cidade ou de estado". (P6)

Apesar da idéia de convivência social integrada veiculada na sociedade globalizada, de uma sociedade influenciada pelas constantes transformações tecnológicas e científicas, a maioria dos Programas de Pós-Graduação em saúde ainda está distanciada dos serviços de saúde e, ao retornar de uma Qualificação e perceber a impossibilidade de mudar a práticas no seu contexto, o profissional se sente desestimulado. Daí decorre a importância periódica das avaliações internas nesses programas.

Conforme apresentado um processo avaliativo deve abranger "o ensino, a formação profissional e cidadã, [...] o destino dos exalunos, a adequação dos critérios de aprovação e promoção de estudantes em seus cursos e de professores na carreira docente, [...] a integração/desintegração entre teoria e prática, entre o Que a universidade produz e as necessidades"(16).

No Que se refere à conceituação dos objetivos propostos no Projeto-Pedagógico do Curso avaliado, obteve-se uma média de conceito igual a 7,37 (sete e trinta e sete), conforme pode ser observado no Quadro 3.

Ao analisar os objetivos propostos e seus respectivos conceitos, constatamos a necessidade de reestruturação do projeto políticopedagógico do curso avaliado de modo que a nova proposta se aproxime mais das mudanças sociais pelas Quais a realidade de saúde vem passando. Essa reestruturação, de fato desencadeada logo após o estudo, seguiu na direção de estabelecer um diálogo permanente entre as potencialidades e as fragilidades identificadas no processo de avaliação contínuo entre discentes e docentes e, periódico, com os egressos.

Os informantes, de modo geral, salientaram a importância da instrumentalização para o mercado de trabalho. Compreendem Que o profissional precisa estar preparado para visualizar a realidade de forma ampla e, ao mesmo tempo, estar inserido na realidade de forma socialmente responsável. Na avaliação dos egressos, o objetivo do mestrado de propor, aplicar e avaliar projetos assistenciais com base em reflexões críticas sobre as práticas de Enfermagem e de saúde, foi considerado como ponto de maior relevância, conforme reflete uma fala a seguir:

“Resolvi fazer o mestrado após uma jornada de 20 anos na assistência, já me encontrando desiludida profissionalmente. $O$ retorno a Academia revigorou o prazer de ser enfermeira, mostrou novas aptidões, reafirmou alguns valores, remodelou 


\begin{tabular}{|lc|}
\hline Itens avaliados & Médias \\
\hline Propor, aplicar e avaliar projetos assistenciais com base em reflexões críticas sobre as práticas de & 8,28 \\
Enfermagem e de saúde & 7,84 \\
Capacitar para atuação na área educacional & 7,60 \\
Realizar pesQuisas e análises em serviços de saúde locais ou regionais & 7,56 \\
Planejar ações de saúde & 7,48 \\
Capacitar para atuação na área assistencial & 7,32 \\
Atuar na gestão de serviços de saúde & 5,52 \\
\hline Realizar pesQuisas e análises em serviços de saúde estaduais ou nacionais & \\
\hline
\end{tabular}

Quadro 3. Avaliação média dos objetivos do curso de Mestrado PEN/UFSC na ótica dos egressos. Florianópolis, 2007.

outros. Enfim a profissional Que entrou, não foi a mesma que saiu, foi bem melhor!" (P8).

Nessa direção, os egressos deixam clara a necessidade de um preparo não somente para a área acadêmica, mas também um preparo Que seja relevante para a área assistencial. Evidenciam que o egresso deve ter um conhecimento diferenciado no Que se refere a sua inserção no fazer reflexivo-crítico, tanto no Que diz respeito ao planejamento como também no assessoramento e avaliação de projetos político-assistenciais relacionados às práticas saúde/cuidado de saúde e enfermagem.

Mesmo que o primeiro objetivo do mestrado tenha sido pontuado como de maior relevância, os demais aspectos como a capacitação para a atuação na área educacional, assistencial, gerencial, bem como para o campo da pesquisa e do planejamento das ações de saúde se mostraram importantes do ponto de vista dos egressos. Percebe-se, com isso, Que o mestrando busca uma noção globalizada, ou seja, uma projeção de dentro para fora, Que remete para uma capacidade bem sucedida de mobilizar e fazer valer o conhecimento e ampliar o espaço de referência dos enfermeiros.

Somado às reflexões já expostas é possível acrescentar Que o mestrado em enfermagem se constitui num espaço de construção importante e apropriado para a consolidação de um conhecimento estimulador e inovador de um saber-fazer enfermagem para o pleno exercício de uma profissão Que preconiza a capacidade de atender as demandas sociais, nas mais diferentes esferas e nos mais diferentes contextos.

Os egressos tornam evidente o desejo e, ao mesmo tempo, a necessidade de realizar pesquisas e análises em serviços de saúde estaduais ou nacionais, ou seja, a necessidade de ampliar o foco de atuação profissional e da importância de considerar uma articulação mais ativa entre a produção do conhecimento e o conhecimento aplicado e divulgado.

O dilema da articulação entre a produção de conhecimento e a sua aplicabilidade, ou entre a prática científica, técnica, política e as alternativas desenvolvidas para o seu equacionamento, fazem parte da história da avaliação de programas como prática

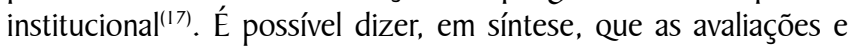
alternativas metodológicas diversas devem fazer parte das preocupações de gestores e/ou responsáveis tanto do setor público como do privado, no sentido de promover a participação e incrementar os processos decisórios dinâmicos e sistemáticos, como importante contribuição para o fortalecimento da responsabilidade e compromisso social.

\section{CONSIDERAÇÕES FINAIS}

O processo de avaliação é, geralmente, um processo complexo, mais especificamente nos programas de pós-graduação, onde estão presentes diversos interesses políticos, ideológicos, desempenho dos cursos, imagem perante a classe, avaliações externas como as promovidas pela CAPES, dentre outros ${ }^{(18)}$. Mas, é fundamental Que este processo seja assumido com seriedade pelas instituições, de modo geral, com o objetivo de confrontar interesses e, principalmente, incrementar o processo interativo e participativo entre os diferentes atores envolvidos.

A importância Que os processos avaliativos vêm adQuirindo na consolidação do sistema de ensino e pesQuisa são decisivos para a promoção e monitoramento da Qualidade e responsabilidade face à missão social. Destaca-se nessa direção, o importante papel Que a CAPES vem desempenhando no sentido da introdução, manutenção e incremento de um sistema de avaliação Que vem contribuindo de forma decisiva para a consolidação - no plano objetivo e subjetivo - de um processo permanente de avaliação dos programas de pós-graduação( ${ }^{(19)}$. Sabe-se Que esse processo, pode, em muito, contribuir para Que os cursos aumentem e mantenham sua Qualidade.

A avaliação da CAPES, no entanto, não pode ser a única via avaliativa. É preciso Que os programas tenham, cada vez mais, uma atitude pró-ativa no sentido de preverem lacunas, visualizarem novas possibilidades e, crescentemente, estimularem a participação dos diferentes atores sociais. Instaurar um processo avaliativo com os egressos se constitui, nessa perspectiva, numa estratégia criativa, inteligente e perspicaz no sentido de detectar fragilidades e antecipar mudanças face às necessidades sociais emergentes.

A avaliação geral possibilita compreender a importância da contribuição ou integração da opinião dos egressos como complemento desse processo, entendendo Que a posição destes possibilita evidenciar suas dificuldades e êxitos, bem como contribuir com novas propostas para a Qualificação dos programas. Compreender a visão dos egressos é contribuir para Que uma outra posição possibilite uma reflexão e amplie as formas de pensar e projetar o futuro dos programas de pós-graduação de enfermagem, principalmente, no sentido de prever e antecipar mudanças ${ }^{(20)}$. 
Assim, importa neste processo, deixar falar, fazer rupturas, problematizar, consolidando a avaliação educacional numa perspectiva de ação social formativa e construtiva com práticas integradoras, mesmo Quando se utiliza de práticas reguladoras.

\section{REFERÊNCIAS}

I. Rodrigues RAP, Erdmann AL, Fernandes JD, Araújo TL. Pósgraduação em enfermagem no Brasil e no Nordeste. Rev Gaúcha Enferm 2007; 28(I): 70-8.

2. Ministério da Educação e Cultura (BR). Secretaria de Educação Superior. Coordenação de Aperfeiçoamento de Pessoal de Nível Superior. I Plano Nacional de Pós-Graduação: 1975-1979. Brasília: CAPES; 1975.

3. Ministério da Educação e Cultura (BR). Secretaria de Educação Superior. Coordenação de Aperfeiçoamento de Pessoal de Nível Superior. II Plano Nacional de Pós-Graduação: 1982-1985. Brasília: CAPES; 1982.

4. Ministério da Educação e Cultura (BR). Secretaria de Educação Superior. Coordenação de Aperfeiçoamento de Pessoal de Nível Superior. III Plano Nacional de Pós-Graduação: 1986- 1989. Brasília: CAPES; 1986.

5. Ministério da Educação e Cultura. Secretaria de Educação Superior. Coordenação de Aperfeiçoamento de Pessoal de Nível Superior. IV Plano Nacional de Pós-Graduação: 2005-2010 Brasília: CAPES; 2005.

6. Ministério da Educação e Cultura (BR). Secretaria de Educação Superior. Coordenação de Aperfeiçoamento de Pessoal de Nível Superior. Plano Nacional de Pós-graduação 2005-20 I 0. Brasília (DF): CAPES, 2005. [citado em 23 set. 2007] Disponível em: http://www.capes.gov.br/sobre/estatísticas.

7. Brasil. Coordenação de Aperfeiçoamento de Pessoal de Nível Superior. Avaliação da Pós-Graduação [citado em 23 set. 2007] Disponível em: http://www.capes.gov.br/avaliacao/ avaliacaopos.html

8. Coordenação de Aperfeiçoamento de Pessoal de Nível Superior. Conferência do Presidente da CAPES. Dr. Jorge Almeida Guimarães [citado em 27 out. 2008]. Disponível em: http:// www.unesp.br/propg/apres_eventos/workshop_saude/ workshop_saude.php

9. Ministério da Educação (BR). Lei 5540, de 28 de Novembro de 1968. Fixa normas de organização e funcionamento do ensino superior e sua articulação com a escola média, e dá outras providências. [citado em 23 set. 2007] Disponível em: http://www.planalto.gov.br/ccivil_03/leis/L5540.htm.

10. Ministério da Educação e Cultura (BR). Secretaria de Educação Superior. Coordenação de Aperfeiçoamento de Pessoal de Nível
Superior. [citado em 23 set. 2007]. Disponível em: http:// servicos.capes.gov.br/projetorelacaocursos/jsp/ arealesDet.jsp?cd_area $=40400000 \&$ grandeArea $=$ CIÊNCIAS\%20DA\%20SAÚDE\&areaConh=ENFERMAGEM

I I. Universidade Federal de Santa Catarina. Programa de PósGraduação em Enfermagem. Visão Geral, Evolução e Tendências do Curso de Mestrado em Enfermagem. [citado em 23 set. 2007]. Disponível em: http://www.nfr.ufsc.br/pen/ conteudo.php?\&sys $=$ bd\&id $=6$

12. Bardin L. Análise de Conteúdo. Lisboa: Edições 70; 1977.

13. Ministério da Educação (BR). Lei 9394, de 20 de Dez. de 1996. Estabelece as diretrizes e bases da educação nacional. [citado em 23 de set. de 2007] Disponível em: http:// portal.mec.gov.br/sesu/arQuivos/pdf/lei9394.pdf.

14. Ministério da Saúde (BR). Lei 8080, de 19 de Setembro de 1990. [citado em 12 out 2008] Disponível em: portal.saude.gov.br/portal/arQuivos/pdf/Lei8080.pdf.

15. Ministério da Saúde (BR). Lei 8I42, de 28 de Dezembro de 1990. [citado em 12 de out. de 2008] Disponível em: portal.saude.gov.br/portal/arQuivos/pdf/Lei81 42.pdf.

16. Dias Sobrinho I, Ristoff DI, (organziadores). Avaliação e compromisso público: A educação superior em debate. Florianópolis: Insular; 2003.

17. Novais HMD. Evaluation of health programs, services and technologies. Rev Saúde Pública 2000; 34(5): 547-9.

18. Rangel LAD, Gomes LFAM, Lins MPE Saul Fuks. Avaliação dos programas de pós-graduação em engenharia da UFRI empregando uma variante desenvolvida do método UTA. Pese Oper 2003; 23(2): 285-99.

19. Kerr-Pontes LRS, Pontes RJS, Bosi MLM, Rigotto RM, Silva RM da, Bezerra Filho JG, Kerr WE. Uma reflexão sobre o processo de avaliação das pós-graduações brasileiras com ênfase na área de saúde coletiva. Physis: Rev Saúde Coletiva 2005; 15(1): 83-94.

20. Paiva AM. Rumos e perspectivas do egresso do Programa de Pós-Graduação Stricto-Sensu em educação PUC-Campinas (1993-2004) [dissertação]. Campinas: Programa de PósGraduação em Educação, Pontifícia Universidade Católica de Campinas; 2006. 